\title{
Polarization effects in hadronic reactions in a GeV region
}

\author{
Vladimir Ladygin* ${ }^{* \dagger}$ \\ Join Institute for Nuclear Research, 141980 Dubna, Russian Federation \\ E-mail: vladyginejinr.ru
}

The major goal of the upgraded Nuclotron facility is to obtain the information on the equation-ofstate for dense nuclear matter playing a key role in the understanding of the collapse supernovae and neutron stars stability. These studies can be performed either in heavy ion collisions or via the short-range few nucleon correlations in deuteron induced reactions. The obtained experimental results and future program with the use of polarized deuteron beam and the internal target station are discussed. The polarization studies for the $N N, N A$ and $d A$ reactions with the extracted deuteron beam at the BM@N setup are proposed. The further extension of the polarization program at BM@N is related with the study of the in-medium modification of the polarization for the strange and multi-strange baryons and the spin alignment for vector mesons decaying in hadronic modes.

XXII International Baldin Seminar on High Energy Physics Problems,

15-20 September 2014

JINR, Dubna, Russia

\footnotetext{
${ }^{*}$ Speaker.

${ }^{\dagger}$ Supported in part by the RFBR under grant $N^{0} 13-02-00101$ a
} 


\section{Introduction}

The Nuclotron at JINR will provide beams of heavy ions with energies up to $6 \mathrm{~A} \cdot \mathrm{GeV}$ for isospin symmetric nuclei, and $4.65 \mathrm{~A} \cdot \mathrm{GeV}$ for $A u$ nuclei. In central heavy-ion collisions at these energies, nuclear densities of about 4 times nuclear matter density can be reached. These conditions are well suited to investigate the equation-of-state (EOS) of dense nuclear matter which plays a central role for the dynamics of core collapse supernovae and for the stability of neutron stars. At the same time, heavy-ion collisions are a rich source of strangeness, and the coalescence of kaons with lambdas or of lambdas with nucleons will produce a vast variety of multi-strange hyperons or of light hypernuclei, respectively. Even the production of light double-hypernuclei or of doublestrange dibaryons is expected to be measurable in heavy-ion collisions at Nuclotron energies. The observation of those objects would represent a breakthrough in our understanding of strange matter, and would pave the road for the experimental exploration of the 3-rd dimension of the nuclear chart [1]. These studies are complimentary to the CBM experimental program at SIS100 [2].

Short range correlations (SRC) of nucleons in nuclei is the subject of intensive theoretical and experimental works during last years. Since SRC have densities comparable to the density in the center of a nucleon which is about $\rho \sim 5 \rho_{0}\left(\rho_{0} \approx 0.17 \mathrm{fm}^{-3}\right)$, they can be considered as the drops of cold dense nuclear matter [3]. These studies explore a new part of the phase diagram and very essential to understand the evolution of neutron stars.

The results obtained at BNL [4], SLAC [5] and JLAB [6, 7] clearly demonstrate that: (i) more than $90 \%$ all nucleons with momenta $k \geq 300 \mathrm{MeV} / c$ belong to $2 \mathrm{~N} \mathrm{SRC}$; (ii) probability for a given proton with momenta $300 \leq k \leq 600 \mathrm{MeV} / c$ to belong to $p n$ correlation is $\sim 18$ times larger than for $p p$ correlations; (iii) probability for a nucleon to have momentum $\geq 300 \mathrm{MeV} / c$ in medium nuclei is $\sim 25 \%$; (iv) 3N SRC are present in nuclei with a significant probability [8]. However, still many open questions persist and further investigations are required both from the experimental and theoretical sides. For instance, the experimental data on the spin structure of $2 \mathrm{~N}(\mathrm{I}=1)$ and $3 \mathrm{~N}$ SRC are almost absent.

The main tools to study SRCs at hadronic facilities can be deuteron structure investigations at large internal momenta allowing to explore $2 \mathrm{~N}$ SRC with $I=0 ;{ }^{3} \mathrm{He}$ structure to understand the role of $2 \mathrm{~N}$ SRC with $I=1$ and $3 \mathrm{~N}$ SRC; nuclei breakup $A(p, p p) X, A(p, p n) X, A(p, p p p) X$ etc. with the detection of few nucleons in the final state. The greate importance is the study of the spin effects in these reactions because the data on the SRCs spin structure are scarce. Nuclotron and NICA will allow to investigate the spin effects for multi-nucleon correlations in a wide energy range.

The spin structure of the $n p$ SRCs has been investigated at JINR via the measurements of the tensor analyzing power $A_{y y}$ in deuteron inclusive breakup at different energies in the wide regions of the $x_{F}$ and transverse proton momentum $p_{T}[9,10,11,12,13]$. The data on the tensor analyzing power $A_{y y}$ obtained in the $A(d, p) X$ reaction at different values of $x_{F} \sim 0.61, \sim 0.67, \sim 0.72$ and $\sim 0.78$ and plotted as a function of the proton transverse momentum $p_{T}$ are shown in left panels a), b), c) and d) in Fig.1, respectively. The figure is taken from ref.[13]. It is seen that the $A_{y y}$ data for different $x_{F}$ are strongly dependent of the transverse momentum of the protons, $p_{T}$. Values of $A_{y y}$ are positive at small $p_{T}$ and monotonously decrease while transverse momentum increasing for all $x_{F}$ values. On the other hand, $A_{y y}$ values change the sign at $p_{T} \sim 600 \mathrm{MeV} / c$ independently 

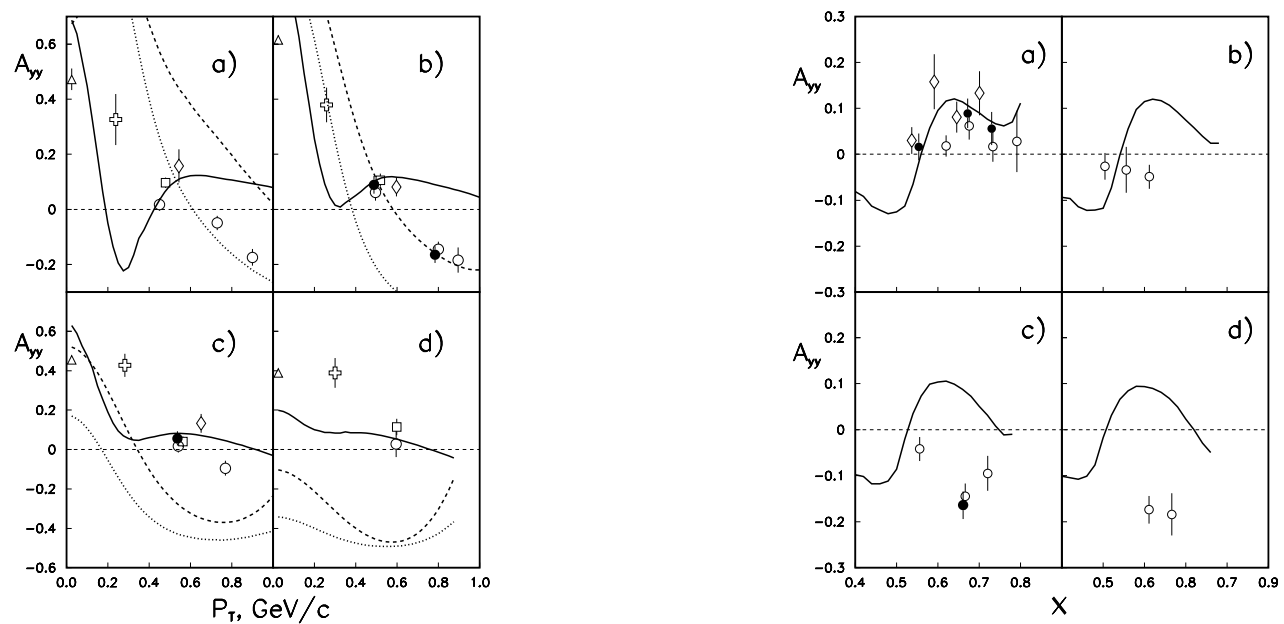

Figure 1: The dependence of the tensor analyzing power $A_{y y}$ as a function of the proton transverse momentum $p_{T}$ at four different fixed values of $x_{F} \sim 0.61,0.67,0.72$ and 0.78, are shown in the a), b), c) and d) left panels, respectively. $A_{y y}$ data plotted as a function of longitudinal momentum fraction $x_{F}$ obtained at fixed $p_{T}$ values of $\sim 550 \mathrm{MeV} / c, \sim 700 \mathrm{MeV} / c, \sim 800 \mathrm{MeV} / c$ and $\sim 900 \mathrm{MeV} / c$ are presented in the a), b), c) and d) right panels, respectively. The data are taken form $[9,10,11,12,13]$. The curves are explained in the text.

on $x_{F}$ and demonstrate kind of negative asymptotic at large $p_{T}$. The dashed, dash-dotted and solid curves are the results of the calculations using standard [14, 15] and covariant [16] deuteron wave functions (DWFs), respectively. In the right panel in Fig.1 the $A_{y y}$ data are plotted at different values of transverse momenta $p_{T}$ as a function of $x_{F}$. The data shown in panels a), b), c) and d) correspond to the averaged values of $p_{T} \sim 550 \mathrm{MeV} / c, \sim 700 \mathrm{MeV} / c, \sim 800 \mathrm{MeV} / c$ and $\sim 900$ $\mathrm{MeV} / c$, respectively. The figure is also taken from ref.[13]. The solid curves are the results of the calculations by using covariant DWF [16]. One can see that the $A_{y y}$ data for different values of $p_{T}$ demonstrate a weak dependence on $x_{F}$. The data obtained at $p_{T} \sim 550 \sim \mathrm{MeV} / c$ are in a good agreement with the calculations by using covariant DWF [16]. At higher $p_{T} A_{y y}$ data have negative values, while the theory predicts a positive sign in the range of measurement Therefore, the $A_{y y}$ data clearly demonstrate the dependence on two internal variables, $x_{F}$ and $p_{T}$ (or their combinations). However, the use of the deuteron structure function that depends on two variables [16] does not allow to describe the data.

The fundamental degrees of freedom in the frame of QCD are the quarks and gluons. These degrees (effective ones as $\Delta \Delta, N^{*} N, N N$ or $6 q$ and $9 q$ components) begin to play a role at the internucleonic distances comparable with the size of the nucleon. At high energies $s$ and large transverse momenta $p_{T}$ the constituent counting rules (CCR) $[17,18]$ are working. These rules predict the dependence of the cross section of the binary re- actions at the fixed scattering angle in the cms as a power-law of $s$. The analysis of the experimental data on the cross sections of the $d p \rightarrow p d$ and $d d \rightarrow^{3}$ Hen reactions [19] has shown that the regime corresponding to CCR can occur already at $T_{d} \sim 500 \mathrm{MeV}$. Therefore, the fundamental degrees of freedom can manifest in the deuteron induced reactions at Nuclotron energies. 


\section{Recent results obtained at internal target}

The main goal of the Deuteron Spin Structure (DSS) experimental program is to obtain the information on the spin - dependent parts of two-nucleon $(2 N)$ and three-nucleon $(3 N)$ forces from two processes: $d p$ - elastic scattering in a wide energy range and $d p$-nonmesonic breakup with two protons detection at energies $300-500 \mathrm{MeV}[20,21,22]$. The motivation of this program is based on theoretical analysis of the experimental results obtained at low and intermediate energies for the deuteron induced reactions (see recent reviews $[23,24]$ and references therein).

Such experimental program at Nuclotron was started by the measurements of the vector $A_{y}$ and tensor $A_{y y}$ and $A_{x x}$ analyzing powers in $d p$ - elastic scattering at $T_{d}$ of $880 \mathrm{MeV}[25]$ and $2000 \mathrm{MeV}$ [26].
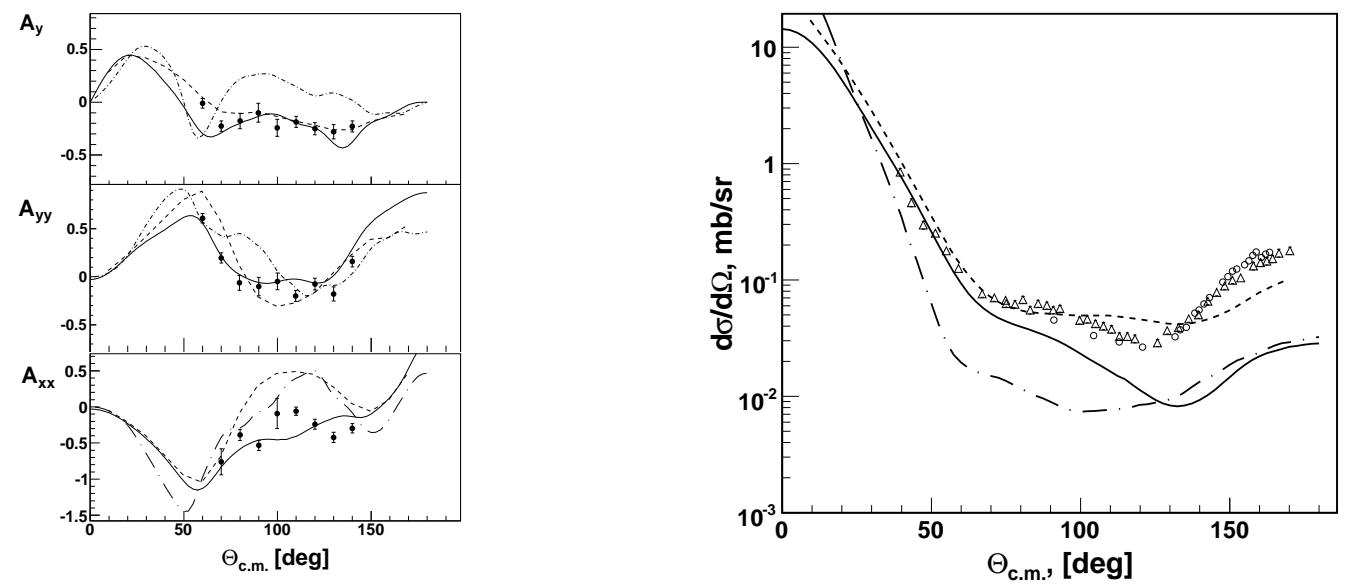

Figure 2: The angular dependence of the analyzing powers $A_{y}, A_{y y}$ and $A_{x x}$ [25] (left panel) and differential cross section [27, 28] (right panel) of $d p$ - elastic scattering at $T_{d} \sim 880 \mathrm{MeV}$. The lines are explained in the text. The pictures are taken from ref. [25].

The results on the angular dependencies of the analyzing powers $A_{y}, A_{y y}$ and $A_{x x}$ of $d p$ - elastic scattering obtained at Nuclotron at $880 \mathrm{MeV}$ [25] are presented in the left panel of Fig.2. The differential cross section obtained at $940 \mathrm{MeV}$ [27] and $850 \mathrm{MeV}$ [28] in the earlier experiments are shown in the right panel of Fig. 2 by the open circles and triangles, respectively. The solid, dashed and dot-dashed lines are the results of the non-relativistic Faddeev calculations [23], relativistic multiple scattering model [29, 30] and optical potential approach [31]. The Faddeev calculations without invoking $3 N$ forces reproduce the behaviour of the analyzing powers, however, they fail to describe the cross section data at the scattering angles larger than $70^{\circ}$ in the cms. The calculations performed within relativistic multiple scattering model $[29,30]$ describes the data on the vector analyzing power $A_{y}$ and cross section. However, there are some problems in the description of the tensor analyzing powers at large angles in the cms. The optical potential approach fails to reproduce both cross section and analyzing powers. The observed deficiencies in the description of the differential cross section and deuteron analyzing powers at $T_{d} \sim 880 \mathrm{MeV}$ obtained at quite large transverse momenta require the consideration of the additional mechanisms, for instance, $3 \mathrm{~N}$ forces. Since present $3 N$ forces models cannot improve the agreement with the data obtained even 
at lower energies, new models of $3 N$ forces (including their short-range part) should be considered. For instance, the next step in the relativistic multiple scattering model [29, 30] development could be taking into account the explicit $\Delta$ - isobar excitation.
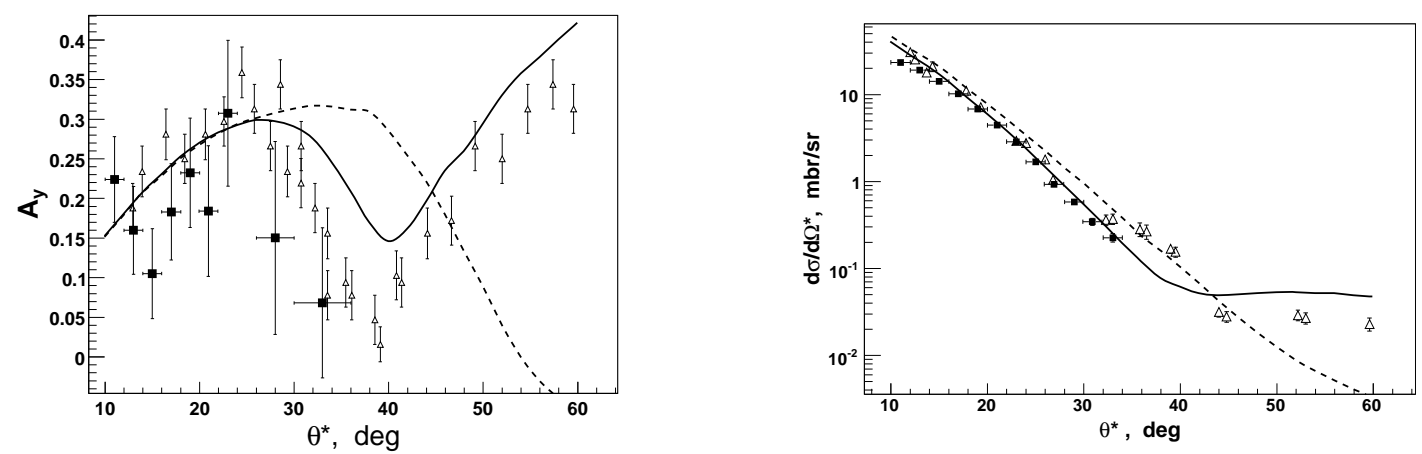

Figure 3: The angular dependence of the analyzing power $A_{y}$ in $d p$ - elastic scattering obtained at $2000 \mathrm{MeV}$ at JINR [32] and ANL [33] is shown in the left panel by the solid squares and open triangles, respectively. The differential cross section data as a function of the scattering angle in the cms obtained at $2000 \mathrm{MeV}$ at JINR [32] and BNL [34] are given in the right panel by the solid squares and open triangles, respectively. The lines are explained in the text. The pictures are taken from ref. [32].

The data on the analyzing power $A_{y}$ in $d p$ - elastic scattering obtained at $2000 \mathrm{MeV}$ at JINR [32] and ANL [33] are shown in the left panel of Fig.3 by the solid squares and open triangles, respectively. The dashed and solid lines are the relativistic multiple scattering model $[29,30]$ calculations without and with double scattering term, respectively. One can see that the single scattering mechanism does not reproduce the experimental data at the scattering angles $\theta^{*}$ larger than $25^{\circ}$. The calculation taking into account the double scattering gives a better agreement with the experimental data in this angular domain.

The right panel of Fig.3 represents the data on the differential cross section in $d p$ - elastic scattering obtained at $2000 \mathrm{MeV}$ at JINR [32] and BNL [34] shown by the solid squares and open triangles, respectively. Meaning of the lines is the same as in the left panel. One can see that inclusion of the double scattering term in the calculations, on the one hand, reduces the value of the cross section in the range of the present measurements and, on the other hand, provides fair agreement with the experimental results up to $\sim 60^{\circ}$ in the $\mathrm{cms}$. Therefore, the data on the cross section and vector analyzing power $A_{y}$ at $2000 \mathrm{MeV}$ are qualitatively described by the relativistic multiple scattering theory $[29,30]$. However, some problems in the description still remain. In this respect new experimental data in deuteron induced reaction are required.

As the first stage of the Deuteron Spin Structure (DSS) experimental program [20, 21, 22] the beam energy scan of $d p$ - elastic scattering cross section at the deuteron energies $400-2000 \mathrm{MeV}$ and measurements of $d p$ - non-mesonic breakup at 300, 400 and $500 \mathrm{MeV}$ in different kinematic configurations have been performed at Nuclotron. These measurements were performed using internal target station at Nuclotron [35] with new control and data acquisition system [36]. The $10 \mu \mathrm{m} \mathrm{CH}_{2}$ foil and $8 \mu \mathrm{m}$ carbon wire were used as the targets. The effect on the hydrogen has been obtained using $\mathrm{CH}_{2}-\mathrm{C}$ subtraction. 
The detection apparatus for the study of $d p$ - elastic scattering consists of 4 scintillation counters with FEU-85 photomultiplier tubes for the detection of the protons and deuterons in coincidence [37]. The amplitudes of the signals and timing information from the detectors were recorded and used in the further data analysis for the $d p$ - elastic scattering events selection. The scintillation counters coupled to Hamamatsu H7416MOD PMTs having better timing and amplitude resolution than FEU-85 PMTs were used for the measurements at $T_{d} \geq 1000 \mathrm{MeV}$. The $d p \rightarrow p p n$ reaction will be investigated using $\Delta E-E$ techniques for the detection of both protons. The details of the experimental setup with $8 \Delta E-E$ detectors are given in ref.[38].

The measurements of the differential cross section of $d p$ - elastic scattering were performed in the energy domain $400-880 \mathrm{MeV}[39,40]$. The data obtained at $500 \mathrm{MeV}, 700 \mathrm{MeV}$ and $880 \mathrm{MeV}$ [39] are in a reasonable agreement with the relativistic multiple scattering theory [29, 30] calculations. The preliminary data on the differential cross section of $d p$ - elastic scattering obtained at Nuclotron at $T_{d} \geq 1000 \mathrm{MeV}$ have been reported in ref.[41]. The data for different kinematic configuration for the $d p \rightarrow p p n$ reaction have been obtained at 300, 400 and $500 \mathrm{MeV}$ [42]. The procedure for useful events selection is described in ref.[43]. The data analysis is in progress.

The continuation of the DSS experimental program is related with new polarized ion source developed at LHEP-JINR [44].

\section{Extracted beam experiments}

\subsection{Spin studies with polarized deuteron beam}

Spin physics with extracted polarized deuteron beam from new polarized deuteron source [44] can be performed with the start version of the BM@N setup [1]. Since the multiplicity of the secondary particles is small ( 2 or 3 tracks) the forward and outer trackers only can be used. The BM@N experimental setup is installed at the 6V beamline in the fixed-target hall of the Nuclotron. The $6 \mathrm{~V}$ beamline contains the quadrupole lenses doublet, two dipole magnets allowing to correct the beam position in the vertical and horizontal planes, and large aperture SP41 dipole magnet for the momentum measurements [1]. The first results with the relativistic deuteron [45] and carbon [46] beams are demonstrated the feasibility of the dense baryonic matter studies with light nuclei using $6 \mathrm{~V}$ beamline infrastructure.

The major direction of possible spin studies is the investigation of the spin-structure of light nuclei at short inter-nucleonic distances in different deuteron induced reactions: $A(d, p) X, d(p, p) d$, $d(d, p) t\left(d\left(d,{ }^{3} \mathrm{He}\right) n\right),{ }^{3} \mathrm{He}(\mathrm{d}, \mathrm{p}){ }^{4} \mathrm{He}[20,21,22]$ etc. in order to obtain independent information on the SRC. Another topics are the investigation of the change of the baryonic resonances properties in nuclear matter via the measurement of the polarization observables $\left(T_{20}\right.$ etc.) in the $A(d, p p) X$ and $A(d, d) X$ reactions; the investigation of the spin effects in the meson production using the $A\left(d,{ }^{3} \mathrm{He}\right) X$ reactions; the investigation of the spin effects in neutron induced reactions (with the proton spectator detection) like $n p \rightarrow p n, n p \rightarrow p p \pi^{-}, n p \rightarrow n p \pi^{+} \pi^{-}, n p \rightarrow d \pi^{+} \pi^{-}$etc.

The transportation line VP1 does not require modification in the sense of the installation or removing of magnetic elements. But spin program realization requires to install the polarimeters (with $\mathrm{CH}_{2}-\mathrm{C}$ targets) at F3 and F5 focuses for polarimetry of the deuteron (or proton) beam. Also the place at F5 (or F3) is needed to install liquid hydrogen (deuterium) target, polarized ${ }^{3} \mathrm{He}$ target 
[47] and set of nuclear targets. Perhaps, it will be necessary to have some place for the stop timeof-flight detector.

The spin physics can be divided on 3 part due to position of the target at VP1 transportation beam line. The physics with the target position at F5(or F3) focus is related with the measurements of the polarization observables in the reactions with the emission of the detected particle at forward angles. The solid angle is defined by the lenses doublet 7k100-8k100 of the VP1 transportation beam line. The separation of the primary deuteron and secondary beams is provided by the bending magnet 3SP40. The physics program can include the measurements

- of the tensor analyzing power $T_{20}$ (and, possibly, vector polarization transfer coefficient $\kappa_{0}$ ) in the inclusive deuteron breakup, $A\left(d, p\left(0^{\circ}\right)\right) X,[48,49,50]$ at the highest available energy at Nuclotron;

- of the tensor analyzing power $T_{20}$ in the inclusive pion production, $A\left(d, \pi^{-}\left(0^{\circ}\right)\right) X$, [51] also at the highest available energy at Nuclotron;

- of the tensor analyzing power $T_{20}$ (and, possibly, vector polarization transfer coefficient $\kappa_{0}$ ) in the inelastic deuteron scattering, $A\left(d, d^{\prime}\right) X$, in the vicinity of the baryonic resonances excitation [52];

- of the tensor analyzing power $T_{20}$ (and, possibly, vector polarization transfer coefficient $\kappa_{0}$ in $p(d, p) d[53,54]$ and $d(d, p) t[55]$ reactions;

- of the tensor analyzing power $T_{20}$ and spin correlation $C_{y, y}$ in the ${ }^{3} \mathrm{He}(d, p){ }^{4} \mathrm{He}$ reaction in the kinetic energy range between 1.0 and $1.75 \mathrm{GeV}[56,57]$;

- of the analyzing power $T_{20}$ in the $A\left(d,{ }^{3} \mathrm{He}\right) X[58]$ reactions.

The measurements of the ${ }^{3} \mathrm{He}(d, p)^{4} \mathrm{He}$ reaction is mostly challenging throughout the above mentioned experiments due to low density of the polarized ${ }^{3} \mathrm{He}$ target [47]. The goal of the ${ }^{3} \mathrm{He}(d, p){ }^{4} \mathrm{He}$ reaction study at Nuclotron is to understand the reasons of the long staying puzzle, namely, the behaviour of the tensor analyzing power $T_{20}$ in $d p$-backward elastic scattering $[53,54]$. While $t_{20}$ data in $e d$ - elastic scattering obtained at JLAB [59] and $T_{20}$ data in $d p$ - inclusive breakup $[48,49]$ can be explained by using the conventional deuteron structure functions and additional to the Born approximation mechanisms, the $T_{20}$ in $d p$-backward elastic scattering demonstrate unexplained the strange structure at the internal momentum $k \sim 0.3-0.5 \mathrm{GeV} / c$ in the vicinity of the $D$ - wave dominance.

The experiments performed at RIKEN at the energies below $270 \mathrm{MeV}$ have shown that the polarization correlation coefficient, $C_{/ /}=1-\frac{1}{2 \sqrt{2}} T_{20}+\frac{3}{2} C_{y, y}$, for the ${ }^{3} \mathrm{He}(d, p)^{4} \mathrm{He}$ reaction may be a unique probe to the D-state admixture in deuteron [56]. The usefulness of this observable to investigate the D-state admixture is attributed to the strong spin-selectivity in neutron capture process by ${ }^{3} \mathrm{He}$ nucleus, i.e., spins of transferred neutron and ${ }^{3} \mathrm{He}$ must be anti-parallel to each other in order to form ${ }^{4} \mathrm{He}$ in the final state. In the one-nucleon exchange (ONE), the expression for $C_{/ /}$is proportional to the $\mathrm{D}$-state fraction in deuteron as

$$
C_{/ /}=\frac{9}{4} \frac{w^{2}}{u^{2}+w^{2}}
$$


where $u$ and $w$ are the $\mathrm{S}$ - and $\mathrm{D}$-state wave functions of deuteron in momentum space. This is a marked contrast to $T_{20}$ and $\kappa_{0}$ for $d p$ backward elastic scattering which include S- and D-state interference term ( $u w$-term) together with a $w^{2}$-term. It is thus expected that $C_{/ /}$may be a candidate to provide an information on the deuteron structure complementary to those from $T_{20}$ and $\kappa_{0}$ obtained in $d p$ - backward elastic scattering [53].
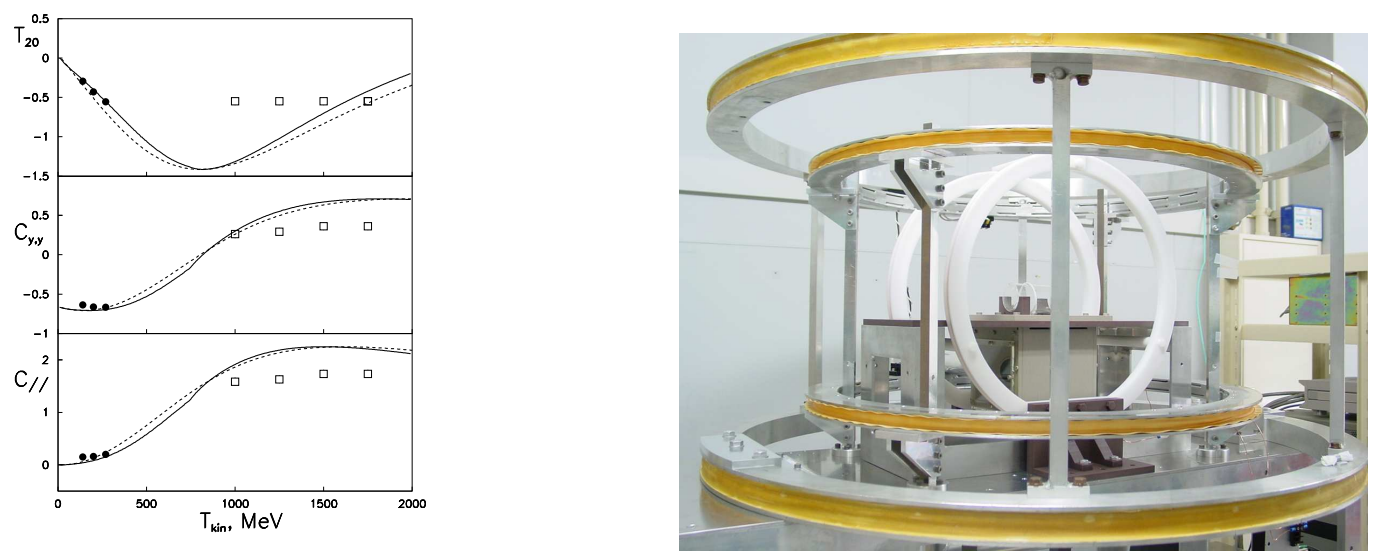

Figure 4: Left panel: tensor analyzing power $T_{20}$, spin correlation $C_{y, y}$ and polarization correlation coefficient $C_{/ /}$for the ${ }^{3} \mathrm{He}(d, p){ }^{4} \mathrm{He}$ reaction. The dashed and solid lines represent one-nucleon exchange calculations without [60] and taking into account the Fermi motion in the target nucleus, respectively. The full symbols are the data obtained at RIKEN [57]. The open squares show the expected precision for the data at Nuclotron. Right panel: modified for Nuclotron experiment polarized ${ }^{3} \mathrm{He}$ target [47].

Tensor analyzing power $T_{20}$, spin correlation $C_{y, y}$ and polarization correlation coefficient $C_{/ /}$ for the ${ }^{3} \mathrm{He}(d, p)^{4} \mathrm{He}$ reaction are shown in the left panel of Fig. 4. Solid lines in the figures represent calculations based on an impulse approximation proposed in Ref. [60]. In the calculation, the Fermi motion in the target nucleus is taken into account [57]. The full symbols are the data obtained at RIKEN [57]. The open squares show the expected precision for the data at Nuclotron.

The main goal of the experiment is to obtain the data on $C_{/ /}$in the energy region of 1.0$1.75 \mathrm{GeV}$, where the contribution from the deuteron D-state is expected to reach a maximum in one-nucleon exchange approximation, to obtain new information on the strange structure observed in the behaviour of $T_{20}$ in the $d p$-backward elastic scattering and to realize experiment on the full determination of the matrix element of the ${ }^{3} \mathrm{He}(d, p)^{4} \mathrm{He}$ reaction in the model independent way. These data will help us also to understand the short-range spin structure of deuteron and effects of non-nucleonic degrees of freedom. For these purposes polarized deuteron beam from new PIS [44] and spin-exchange-type polarized ${ }^{3} \mathrm{He}$ target developed at CNS of Tokyo University [47] and modified for the experiment at Nuclotron can be used.

The polarization observables in the $p(d, p) d[53,54]$ and $d(d, p) t[55,61,62]$ reactions in the collinear geometry can be measured with the same experimental setup using liquid hydrogen or deuterium target or $\mathrm{CH}_{2}$ and $C D_{2}$ solid targets with carbon background subtraction.

The solid nuclear target can be placed inside the pole of the 3SP40 magnet. It can be varied from the beginning to the middle of the pole of the 3SP40 magnet. The target position in the middle 
of the switched off magnet corresponds to $\sim 100 \mathrm{mr}$. The following experiments can be performed in this configuration:

- of the tensor $A_{y y}$ and vector $A_{y}$ analyzing powers (and, possibly, vector polarization transfer coefficient $\left.C_{y}^{y}\right)$ in inclusive deuteron breakup, $A(d, p) X$, at large transverse proton momenta $[9,10,11,12,13]$ at the highest available energy at Nuclotron;

- of the tensor $A_{y y}$ and vector $A_{y}$ analyzing powers [63, 64, 65, 66] (and, possibly, vector polarization transfer coefficient $\left.C_{y}^{y}[67]\right)$ in the inelastic deuteron scattering, $A\left(d, d^{\prime}\right) X$, in the vicinity of the baryonic resonances excitation;

- of the tensor $A_{y y}$ and vector $A_{y}$ analyzing powers in the inclusive pion production, $A\left(d, \pi^{-}\right) X$, [68] also at the highest available energy at Nuclotron.

The measurements of the analyzing powers in inclusive deuteron breakup and the inelastic deuteron scattering in the vicinity of the baryonic resonances excitation can be performed simultaneously. The measurements of the cumulative $\pi^{-}$production will require the change of the polarity in the magnetic elements of $6 \mathrm{~V}$ beam line. All these experiments require the additional TOF detector placed between F5 and F6 focuses.

The third part of the measurements is the spin physics with the target position at F6 focus. The position of the F6 focus can be change by the magnetic optics of the $6 \mathrm{~V}$ beam line. The target position for this part of the experiments can be varied depending on the readiness of the inner tracker. For the low multiplicity events ( 2 or 3 tracks) one can use the same forward tracker as for the discussed above experiments. The separation of the primary deuteron and secondary beams is provided by the modernized analyzing magnet SP41 [69]. The physiscs is related with the baryonic resonances spin properties studies at the energies between 2 and $6 \mathrm{GeV}$ of the deuteron kinetic energy and includes the measurements:

- of the tensor $A_{y y}$ and vector $A_{y}$ analyzing powers in quasi-elastic and inelastic $A(d, p p) X$ reaction [70];

- of the tensor $A_{y y}$ and vector $A_{y}$ analyzing powers in the inelastic deuteron scattering $A\left(d, d^{\prime}\right) X$ [67] and $A\left(d, d^{\prime}\right) \pi^{ \pm} X$ reactions;

- investigation of the vector analyzing power in neutron induced reactions (with the proton spectator detection) like $n p \rightarrow p n, n p \rightarrow p p \pi^{-}, n p \rightarrow n p \pi^{+} \pi^{-}[71]$ etc.

For the these experiments the full size RPC wall is required. In parallel, the measurement of the $d p$ - elastic scattering [25, 26, 72], $d d \rightarrow{ }^{3} \mathrm{He} n[73,74,75]$ and $d A \rightarrow{ }^{3} \mathrm{He} X$ [58] processes can be performed.

The part of the spin physics program with polarized deuterons require to use only magnet, outer tracker, forward tracker based on straw or scintillation fiber hodoscopes, and part of the RPC wall. As the start time-of-flight counter one can use scintillation or cherenkov counters. In addition the development of movable liquid hydrogen(deuterium) target of $5-10 \mathrm{~cm}$ along the beam is required. During last years a significant progress in the preparation of the BM@N experiment has been achieved. The measurements with 1.0-4.0 A. GeV deuteron [45] and 3.42 A.GeV carbon 
[46] beams demonstrated the feasibility of the experiment with the existing beamlines. On the base of these measurements the technical requirements to the Nuclotron parameters, beam transportation conditions and experimental cave details were formulated. The experimental zone and detectors are in preparation.

The spin studies with BM@N requires also the advanced deuteron beam polarimetry at $\mathrm{Nu}$ clotron discussed in [76, 77].

\subsection{Polarization effects in heavy ion collisions}

New signature investigated at BM@N [78] can be the change of in the polarization properties of the secondary particles in the nucleus-nucleus collisions compared to the nucleon-nucleon collisions. A number of polarization observables have been proposed as a possible signature of phase transition, namely, decreasing of the $\Lambda^{0}$ transverse polarization in central collisions [79, 80, 81], non-zero $\bar{\Lambda}^{0}$ longitudinal polarization [82, 83], non-zero $J / \Psi$ polarization at low $p_{T}$ [84], anisotropy in dielectron production from vector mesons decay [85], global hyperon polarization [86] and spin-alignment of vector mesons [87] in non-central events etc. The study of the modification of the $\Lambda^{0}$ transverse polarization and global $\Lambda^{0}$ polarization at NICA and FAIR energies has been proposed in ref. [88]. Recently the vorticity and hydrodynamical helicity in noncentral heavyion collisions were studied for Nuclotron/NICA energies as the functions of the energy collision, system size etc.[89].

\section{Conclusions}

New data on the analyzing powers $A_{y}, A_{y y}$ and $A_{x x}$ in $d p$ - elastic scattering at various energies up to $2000 \mathrm{MeV}$ and well as for the $d p$-nonmesonic breakup at the energies between 300 and 500 $\mathrm{MeV}$ for different kinematic configurations can be measured at ITS at the Nuclotron.

First stage of the BM@N setup (without or with reduced version of the inner tracker) is well suited for the physics with polarized deuterons using new PIS [44].

Measurements of the polarization effects in the heavy ion collisions can significantly enrich the physics at BM@N.

\section{References}

[1] V. Ladygin et al., Study of strange matter production in the heavy ion collisions at NUCLOTRON, in proceedings of the XXI-st International Baldin Seminar on High Energy Physics Problems:

Relativistic Nuclear Physics and Quantum Chromodynamics (ISHEPP 2012), 10-15 September, 2012, Dubna, Russia, PoS (Baldin-ISHEPP-XXI) 038 (2012).

[2] B. Friman, C. Hohne, J. Kroll, S. Leupold, J.Randrup, R. Rapp and P. Senger, The CBM physics book: Compressed baryonic matter in laboratory experiments, Lect.Notes Phys. 814 (2011) 1.

[3] L. Frankfurt, M. Sargsian, M. Strikman, Recent observation of short range nucleon correlations in nuclei and their implications for the structure of nuclei and neutron stars, Int.J.Mod.Phys. A23 (2008) 2991.

[4] E. Piasetzky, M. Sargsian, L. Frankfurt, M. Strikman, J.W. Watson, Evidence for the strong dominance of proton-neutron correlations in nuclei, Phys.Rev.Lett. 97 (2006) 162504. 
[5] L.L. Frankfurt, M.I. Strikman, D.B. Day, M.M. Sargsian, Evidence for short range correlations from high $Q^{2}\left(e, e^{\prime}\right)$ reactions, Phys.Rev. C48 (1993) 2451.

[6] K.Sh. Egiyan et al., Observation of nuclear scaling in the $A\left(e, e^{\prime}\right)$ reaction at $x(B)$ greater than 1 , Phys.Rev. C68 (2003) 014313.

[7] K.S. Egiyan et al., Measurement of 2- and 3-nucleon short range correlation probabilities in nuclei, Phys.Rev.Lett. 96 (2006) 082501.

[8] L. Frankfurt, M. Sargsian, and M. Strikman, Future directions for probing two and three nucleon short-range correlations at high energies AIP Conf.Proc. 1056, 322 (2008).

[9] L.S. Azhgirey et al., Measurement of the tensor analyzing power $T_{20}$ in inclusive deuteron breakup at 9-GeV/c on hydrogen and carbon, Phys.Lett. B387 (1996) 37.

[10] S.V. Afanasev et al., Measurement of the tensor analyzing power $A_{y y}$ in inclusive breakup of 9 GeV/c deuterons on carbon at large transverse momenta of protons, Phys.Lett. B434 (1998) 21;

L.S. Azhgirey et al., Differential cross-section for the reaction ${ }^{12} C(d, p) X$ at a primary momentum of $9 \mathrm{GeV/c}$ and its tensor and vector analyzing powers, Phys.Atom.Nucl. 62 (1999) 1673.

[11] V.P. Ladygin et al.,Measurement of the tensor-analyzing power $A_{y y}$ in deuteron breakup at $4.5 \mathrm{GeV/c}$ and 80 mr, Few Body Syst. 32 (2002) 127;

L.S. Azhgirey et al., Measurement of the differential cross-section, vector and tensor analyzing powers of the $4.5 \mathrm{GeV} / \mathrm{c}$ deuteron breakup on ${ }^{9} \mathrm{Be}$ with the proton emission at $80 \mathrm{mrad}$, Phys.Atom.Nucl. 66 (2003) 690.

[12] L.S. Azhgirey et al., New data on the tensor analyzing power $A_{y y}$ of the relativistic breakup as additional test of deuteron structure at small distances, Phys.Lett. $\mathbf{B 5 9 5}$ (2004) 151.

L.S. Azhgirey et al., Measurement of the tensor $A_{y y}$ and vector $A_{y}$ analyzing powers of the deuteron inelastic scattering off berillium at $5.0 \mathrm{GeV/c}$ and $178 \mathrm{mr}$, Phys.Atom.Nucl. 68 (2005) 991-998

[13] V.P. Ladygin et al., Tensor analyzing power $A_{y y}$ in deuteron inclusive breakup on hydrogen and carbon at $9 \mathrm{GeV/c}$ and large proton transverse momenta, Phys.Lett. B629 (2005) 60;

L.S. Azhgirey et al., Measurement of the tensor $A_{y y}$ and vector $A_{y}$ analyzing powers in the fragmentation of a $9 \mathrm{GeV/c}$ deuteron on hydrogen and carbon nuclei at high proton transverse momenta, Phys.Atom.Nucl. 71 (2008) 264.

[14] R. Machleidt, The high precision, charge dependent Bonn nucleon-nucleon potential (CD-Bonn), Phys.Rev. C63 (2001) 024001.

[15] M. Lacombe, B. Loiseau, R. Vinh Mau, J. Cote, P. Pires and R. de Tourreil, Parametrization of the deuteron wave function of the Paris N-N potential, Phys.Lett. B101 (1981) 139.

[16] V.A. Karmanov and A.V. Smirnov, Electromagnetic form-factors in the light front dynamics, Nucl.Phys. A546 (1992) 691; Deuteron electromagnetic form-factors in the light front dynamics, Nucl.Phys. A575 (1994) 520;

J. Carbonell and V.A. Karmanov, Relativistic deuteron wave function in the light front dynamics, Nucl.Phys. A581 (1995) 625; Relativistic wave function of the np system in the $J(\pi)=0^{+}$continuous spectrum state, Nucl.Phys. 589 (1995) 713;

J. Carbonell, B. Desplanques, V.A. Karmanov and J.F. Mathiot, Explicitly covariant light front dynamics and relativistic few body systems, Phys.Rep. 300 (1998) 125. 
[17] S.J. Brodsky and G.R. Farrar, Scaling Laws at Large Transverse Momentum, Phys.Rev.Lett. 31 (1973) 1153; Scaling Laws for Large Momentum Transfer Processes, Phys.Rev. D11 (1975) 1309;

G.P. Lepage and S.J. Brodsky, Exclusive Processes in Perturbative Quantum Chromodynamics, Phys.Rev. D22 (1980) 2157.

[18] V.A. Matveev, R.M. Muradyan, and A.N. Tavkhelidze, Automodellism in the large - angle elastic scattering and structure of hadrons, Lett. Nuovo Cim. 7 (1973) 719.

[19] Yu.N. Uzikov, Indication of asymptotic scaling in the reactions $d d \rightarrow p^{3} H, d d \rightarrow n^{3} H e$ and $p d \rightarrow p d$, JETP. Lett. 81 (2005) 303.

[20] V.P. Ladygin et al., Recent results with polarized deuterons and polarimetry at Nuclotron-NICA, J.Phys.Conf.Ser. 295 (2011) 012131.

[21] V.P. Ladygin et al., Spin physics in few body systems at Nuclotron, Phys.Part.Nucl. 45 (2014) 327.

[22] V.P. Ladygin et al., Few-body Studies at Nuclotron-JINR, Few Body Syst. 55 (2014) 709.

[23] W. Glöckle, H. Witala, D. Hüber, H. Kamada, J. Golak, The Three nucleon continuum: achievements, challenges and applications, Phys.Rept. 274 (1996) 107.

[24] N. Kalantar-Nayestanaki, E. Epelbaum, J.G. Messchendorp and A. Nogga, Signatures of three-nucleon interactions in few-nucleon systems, Rept.Prog.Phys. 75 (2012) 016301.

[25] P.K. Kurilkin et al., Measurement of the vector and tensor analyzing powers for dp-elastic scattering at $880 \mathrm{MeV}$, Phys.Lett. B715 (2012) 61.

[26] P.K. Kurilkin et al., Investigation of the angular dependence of the analyzing powers in the deuteron-proton elastic scattering at the nuclotron, Phys.Part.Nucl.Lett. 8 (2011) 1081.

[27] J.C. Alder et al., Elastic pd scattering at 316, 364, 470, and $590 \mathrm{MeV}$ in the backward hemisphere, Phys.Rev. C6 (1972) 2010.

[28] N.E. Booth at al., Proton-deuteron elastic scattering at $1.0 \mathrm{GeV} / \mathrm{c}$, Phys.Rev. D4 (1971) 1261.

[29] N.B. Ladygina, Deuteron-proton elastic scattering at intermediate energies, Phys.Atom.Nucl. 71 (2008) 2039.

[30] N.B. Ladygina, Differential cross section of $d p$ - elastic scattering at intermediate energies, Eur.Phys.J. A42 (2009) 91.

[31] M.A. Shikhalev, Elastic Nd scattering at intermediate energies as a tool for probing the short-range deuteron structure, Phys.Atom.Nucl. 72 (2009) 588.

[32] V.V. Glagolev, V.P. Ladygin, N.B. Ladygina and A.A. Terekhin, Measurement of the differential cross-section and deuteron vector analyzing power in dp-elastic scattering at $2.0 \mathrm{GeV}$, Eur.Phys.J. A48 (2012) 182.

[33] M. Haji-Saeid et al., Tensor and vector spin observables in pd elastic scattering at $600 \mathrm{MeV}$, $800 \mathrm{MeV}$, and $1000 \mathrm{MeV}$, Phys.Rev. C36 (1987) 2010.

[34] G.W. Bennett et al., Proton-deuteron scattering at 1 BeV, Phys.Rev.Lett. 19 (1967) 387.

[35] A.I. Malakhov et al., Potentialities of the internal target station at the Nuclotron, Nucl.Instrum.Meth. in Phys.Res. A440 (2000) 320.

[36] A.Yu. Isupov, V.A. Krasnov, V.P. Ladygin, S.M. Piyadin, S.G. Reznikov, The Nuclotron internal target control and data acquisition system, Nucl.Instrum.Meth. in Phys.Res. A698 (2013) 127. 
[37] Yu.V. Gurchin et al., Detection equipment for investigating dp elastic scattering at internal target of nuclotron in the framework of DSS project, Phys.Part.Nucl.Lett. 8 (2011) 950.

[38] S.M. Piyadin et al., $\Delta E-E$ detector for proton registration in nonmesonic deuteron breakup at the Nuclotron internal target, Phys.Part.Nucl.Lett. 8 (2011) 107.

[39] Yu.V. Gurchin et al., The cross-section in dp-elastic scattering at the energies of $500 \mathrm{MeV}, 700 \mathrm{MeV}$ and $880 \mathrm{MeV}$ obtained at the internal target station of Nuclotron, Phys.Part.Nucl.Lett. 10 (2013) 243.

[40] Yu.V. Gurchin et al., The differential cross-section on dp-elastic scattering at 400-880 MeV obtained at Nuclotron, Nucl.Phys.Proc.Suppl. 245 (2013) 271.

[41] A.A. Terekhin et al., Study of dp-elastic scattering at energies 650, 750 and $1000 \mathrm{MeV/nucleon,}$ Nucl.Phys.Proc.Suppl. 245 (2013) 185.

[42] S.M. Piyadin et al., Experiment on the study of the $d p \rightarrow$ ppn reaction at 300-500 MeV of the deuteron energy at ITS at Nuclotron, Nucl.Phys.Proc.Suppl. 245 (2013) 177.

[43] M. Janek, B. Trpisova, S.M. Piyadin and V.P. Ladygin, GEANT4 simulation of dp non-mesonic breakup reaction at 300 and 500 MeV, Phys.Part.Nucl.Lett. 11 (2014) 552.

[44] V.V. Fimushkin et al., Source of polarised deuterons, Eur.Phys.J.ST 162 (2008) 275.

[45] A.A. Terekhin et al., Preparation of experiments to study light nuclei structure at Nuclotron, in proceedings of the XXI-st International Baldin Seminar on High Energy Physics Problems:

Relativistic Nuclear Physics and Quantum Chromodynamics (ISHEPP 2012), 10-15 September, 2012, Dubna, Russia, PoS (Baldin-ISHEPP-XXI) 005 (2012).

[46] S.M. Piyadin et al., First extraction of the $3.42 \mathrm{~A} \cdot \mathrm{GeV}{ }^{12} \mathrm{C}$ beam for studies of baryonic matter at Nuclotron, Phys. Part. Nucl. Lett. 9 (2012) 589.

[47] T. Uesaka et al., Polarized ${ }^{3}$ He project at RIKEN, Nucl.Instr. and Meth. in Phys.Res. A402 (1998) 212.

[48] V. Punjabi et al., Deuteron breakup at $2.1 \mathrm{GeV}$ and $1.25 \mathrm{GeV}$, Phys.Rev. C39 (1989) 608.

[49] L.S. Azhgirey et al., Measurement of the tensor analyzing power $T_{20}$ in inclusive deuteron breakup at $9 \mathrm{GeV/c}$ on hydrogen and carbon, Phys.Lett. B387 (1996) 37.

[50] B. Kuhn et al., The measurements of the polarization transfer coefficient in the $(d, p)$ reaction at a fixed proton momentum $4.5 \mathrm{GeV/c}$ and a deuteron momentum range of $6 \mathrm{GeV} / \mathrm{c}$ to $9 \mathrm{GeV/c}$, Phys.Lett. B334 (1994) 298-303

[51] S. Afanasev et al., Fragmentation of tensor polarized deuterons into cumulative pions, Phys.Lett. B445 (1998) 14.

[52] L.S. Azhgirey et al., First measurement of the tensor analyzing power, $T_{20}$, in inelastic $\left(d, d^{\prime}\right) X$ scattering at $0^{\circ}$ on ${ }^{1} \mathrm{H}$ and ${ }^{12} \mathrm{C}$ at $4.5 \mathrm{GeV} / \mathrm{c}$ and $5.5 \mathrm{GeV} / \mathrm{c}$, Phys.Lett. $\mathrm{B361}$ (1995) 21.

[53] V. Punjabi et al., Measurement of polarization transfer $\kappa_{0}$ and tensor analyzing power $T_{20}$ in the backward elastic dp scattering, Phys.Lett. B350 (1995) 178.

[54] L.S. Azhgirey et al., Tensor analyzing power $T_{20}$ in backward elastic dp scattering and breakup at $0^{\circ}$ between $3.5 \mathrm{GeV/c}$ and $6.5 \mathrm{GeV} / \mathrm{c}$, Phys.Lett. B391 (1997) 22.

[55] V.P. Ladygin et al., Measurement of the tensor analyzing power $T_{20}$ in the $d d \rightarrow{ }^{3} \mathrm{Hen}$ and $d d \rightarrow{ }^{3} \mathrm{H} p$ at intermediate energies and at zero degree, Phys.Lett. B598 (2004) 47.

[56] T. Uesaka et al., Polarization correlation coefficient for the ${ }^{3} \mathrm{He}(\vec{d}, p){ }^{4} \mathrm{He}$ reaction, Phys.Lett. B467 (1999) 199. 
[57] T. Uesaka et al., ${ }^{3} \mathrm{He}(d, p){ }^{4} \mathrm{He}$ reaction at intermediate energies and impulse picture of the $(d, p)$ reactions, Phys. Lett. $\mathbf{B 5 3 3}$ (2002) 1.

[58] V.P. Ladygin and N.B. Ladygina, Polarization observables for the collinear $d p \rightarrow{ }^{3} \mathrm{He} \pi^{0}$ reaction, Phys.Atom.Nucl. 58 (1995) 1283.

[59] D. Abbott et al., Measurement of tensor polarization in elastic electron deuteron scattering at large momentum transfer, Phys.Rev.Lett. 84 (2000) 5053.

[60] H. Kamada et al., A model for the ${ }^{3} \mathrm{He}(\vec{d}, p)^{4} \mathrm{He}$ reaction at intermediate-energies, Prog.Theor.Phys. 104 (2000) 703.

[61] N.B. Ladygina, $d d \rightarrow{ }^{3}$ Hen reaction at intermediate energies, Few Body Syst. 53 (2012) 253.

[62] N.B. Ladygina, Spin effects in the $d d \rightarrow{ }^{3} \mathrm{Hen}$ reaction at intermediate energies, Phys.Part.Nucl. 45 (2014) 187.

[63] V.P. Ladygin et al., Measurement of the tensor analyzing power $A_{y y}$ in the inelastic scattering of deuterons in the vicinity of excitation of baryonic resonances, Eur.Phys.J. A8 (2000) 409.

[64] L.S. Azhgirei et al, Measurement of the tensor analyzing power $A_{y y}$ in the inelastic scattering of $4.5 \mathrm{GeV} / \mathrm{c}$ deuterons on beryllium at an angle of $80 \mathrm{mrad}$, Phys.Atom.Nucl. 64 (2001) 1961.

[65] L.S. Azhgirey et al., Measurement of the tensor $A_{y y}$ and vector $A_{y}$ analyzing powers of the deuteron inelastic scattering off berillium at $5.0 \mathrm{GeV} / \mathrm{c}$ and $178 \mathrm{mr}$, L.S. Azhgirey Phys.Atom.Nucl. 68 (2005) 991.

[66] V.P. Ladygin. et al., Tensor $A_{y y}$ and vector $A_{y}$ analyzing powers in the $H\left(d, d^{\prime}\right) X$ and ${ }^{12} C\left(d, d^{\prime}\right) X$ reactons at initial deuteron momenta of $9 \mathrm{GeV/c}$ in the region of baryonic resonances excitation, Phys.Atom.Nucl. 69 (2006) 852.

[67] V.P. Ladygin and N.B. Ladygina, Polarization effects in inelastic deuteron scattering in the region of baryon-resonance excitation, Phys.Atom.Nucl. 65 (2002) 182.

[68] L.S. Azhgirey et al., Investigation of the deuteron spin structure at short nucleon-nucleon distances in the reaction of polarized-deuteron fragmentation to cumulative pions, Phys.Atom.Nucl. 74 (2011) 1392.

[69] P.G. Akishin et al., Optimization of a large aperture dipole magnet for baryonic matter studies at Nuclotron, e-Print: arXiv:1407.7096 [physics.ins-det], to be published in Phys.Part.Nucl.Lett. (2015).

[70] T. Sams et al.,Quasifree $\left(\vec{d}^{2}{ }^{2}\right.$ He $)$ data, Phys.Rev. C51 (1995) 1945.

[71] A.K. Kurilkin et al., Double pion production in np and pp collisions at $1.25 \mathrm{GeV}$ with HADES, in proceedings of the XXI-st International Baldin Seminar on High Energy Physics Problems: Relativistic Nuclear Physics and Quantum Chromodynamics (ISHEPP 2012), 10-15 September, 2012, Dubna, Russia, PoS (Baldin-ISHEPP-XXI) 041 (2012).

[72] P.K. Kurilkin et al., New data on the differential cross section of the dp-elastic scattering at $2.5 \mathrm{GeV}$ obtained with HADES detector, in proceedings of the XXI-st International Baldin Seminar on High Energy Physics Problems: Relativistic Nuclear Physics and Quantum Chromodynamics (ISHEPP 2012), 10-15 September, 2012, Dubna, Russia, PoS (Baldin-ISHEPP-XXI) 040 (2012).

[73] M. Janek et al., Analyzing powers $A_{y y}, A_{x x}, A_{x z}$ and $A_{y}$ in the $d d \rightarrow{ }^{3} \mathrm{Hen}$ reaction at $270 \mathrm{MeV}$, Eur.Phys.J. A33 (2007) 39.

[74] A.K. Kurilkin et al., The angular distributions of the vector $A_{y}$ and tensor $A_{y y}, A_{x x}, A_{x z}$ analyzing powers in the $d d \rightarrow{ }^{3} \mathrm{Hp}$ and $d d \rightarrow{ }^{3} \mathrm{Hen}$ reactions at $E_{d}=200 \mathrm{MeV}$ and $270 \mathrm{MeV}$, Int.J.Mod.Phys. A24 (2009) 526. 
[75] A.K. Kurilkin et al., Angular distributions of the vector $A_{y}$ and tensor $A_{y y}, A_{x x}, A_{x z}$ analyzing powers in the $d d \rightarrow{ }^{3} \mathrm{H}$ p reaction at $200 \mathrm{MeV}$, Phys.Rev. C87 (2013) 051001(R).

[76] P.K. Kurilkin et al., The $270 \mathrm{MeV}$ deuteron beam polarimeter at the Nuclotron Internal Target Station, Nucl.Instr.Meth. in Phys.Res. A642 (2011) 45.

[77] P.K. Kurilkin and V.P. Ladygin, Deuteron beam polarimetry at the Nuclotron, Phys.Part.Nucl. 45 (2014) 265.

[78] V. Ladygin et al., Experimental program for baryonic matter studies, in proceedings of the $X V$-th Advanced Research Workshop on High Energy Spin Physics (DSPIN-13), 8-12 October 2013, Dubna, Russia; Edited by A.V. Efremov and S.V. Goloskokov, JINR, Dubna, ISBN 978-5-9530-0315-3 (2014) 239.

[79] A.D. Panagiotou, $\Lambda^{0}$ nonpolarization: possible signature of quark matter, Phys.Rev. C33 (1986) 1999.

[80] A. Ayala, E. Cuautle, G. Herrera and L.M. Montano, $\Lambda^{0}$ polarization as a probe for production of deconfined matter in ultrarelativistic heavy ion collisions, Phys.Rev. $\mathbf{C 6 5}$ (2002) 024902.

[81] A.Ya. Berdnikov et al., Polarization of $\Lambda^{0}$ hyperons as a signature for the quark gluon plasma, Acta Phys. Hungary. A22 (2005) 139.

[82] M. Jacob, $\Lambda(\bar{\Lambda})$ longitudinal polarization: a signature for the formation of a quark - gluon plasma in heavy ion collisions, Z.Phys. C38 (1988) 273.

[83] G. Herrera, G. Magnin and L.M. Montano, Longitudinal $\bar{\Lambda}^{0}$ polarization in heavy ion collisions as a probe for QGP formation, Eur.Phys.J. C39 (2005) 95.

[84] B.L. Ioffe and D.E. Kharzeev, Quarkonium polarization in heavy ion collisions as a possible signature of the quark gluon plasma, Phys.Rev. C68 (2003) 061902(R).

[85] E.L. Bratkovskaya et al., Anisotropy of dilepton emission from nuclear collisions, Phys.Lett. B348 (1995) 283; Anisotropy of dilepton emission from nucleon-nucleon interactions], Phys.Lett. $\mathbf{B 3 4 8}$ (1995) 325; Decay anisotropy of $e^{+} e^{-}$sources from $p N$ and pd collisions, Phys.Lett. B362 (1995) 17; Dilepton anisotropy from $\mathrm{p}+\mathrm{Be}$ and $\mathrm{Ca}+\mathrm{Ca}$ collisions at BEVALAC energies, Phys.Lett. $\mathbf{B 3 7 6}$ (1996) 12; Probing hadronic polarizations with dilepton anisotropies, Z.Phys. C75 (1997) 119.

[86] Z.-T. Liang and X.-N. Wang, Globally polarized quark-gluon plasma in non-central A $+A$ collisions,Phys.Rev.Lett. 94 (2005) 102301, Erratum-ibid. 96 (2006) 039901.

[87] Z.-T. Liang and X.-N. Wang, Spin alignment of vector mesons in non-central A + A collisions, Phys.Lett. B629 (2005) 20.

[88] V.P. Ladygin, A.P. Jerusalimov and N.B. Ladygina, Polarization of $\Lambda^{0}$ hyperons in nucleus-nucleus collisions at high energies, Phys.Part.Nucl.Lett. 7 (2010) 349.

[89] M. Baznat, K. Gudima, A. Sorin and O. Teryaev, Helicity separation in heavy-ion collisions, Phys.Rev. C88 (2013) 061901. 